\title{
On the marginal instability of linear switched systems ${ }^{\text {th }}$
}

\author{
Yacine Chitour ${ }^{\mathrm{a}}$, Paolo Mason ${ }^{\mathrm{a}}$, Mario Sigalotti ${ }^{\mathrm{b}, *}$ \\ ${ }^{a}$ Laboratoire des signaux et systèmes, Université Paris-Sud, CNRS, Supélec, Gif-Sur-Yvette, France \\ ${ }^{\mathrm{b}}$ INRIA Saclay - Île-de-France, Team GECO and CMAP, École Polytechnique, Palaiseau, France
}

\section{A R T I C L E I N F O}

\section{Article history:}

Received 20 March 2011

Received in revised form

25 January 2012

Accepted 2 April 2012

Available online 16 May 2012

\section{Keywords:}

Switched systems

Marginal instability

Barabanov norm

Joint spectral radius

\begin{abstract}
A B S T R A C T
Stability properties for continuous-time linear switched systems are at first determined by the (largest) Lyapunov exponent associated with the system, which is the analogue of the joint spectral radius for the discrete-time case. The purpose of this paper is to provide a characterization of marginally unstable systems, i.e., systems for which the Lyapunov exponent is equal to zero and there exists an unbounded trajectory, and to analyze the asymptotic behavior of their trajectories. Our main contribution consists in pointing out a resonance phenomenon associated with marginal instability. In the course of our study, we derive an upper bound of the state at time $t$, which is polynomial in $t$ and whose degree is computed from the resonance structure of the system. We also derive analogous results for discrete-time linear switched systems.
\end{abstract}

(C) 2012 Elsevier B.V. All rights reserved.

\section{Introduction}

We consider linear switched systems of the form

$\dot{x}(t)=A(t) x(t)$,

where $x \in \mathbb{R}^{n}$ and the switching law $A(\cdot)$ is an arbitrary measurable function taking values on a compact and convex set $\mathcal{A}$ of $n \times n$ matrices. In the following, a switched system of the form (1) will be often identified with the corresponding set of matrices $\mathcal{A}$. This paper is concerned with stability issues for (1), where the stability properties are assumed to be uniform with respect to the switching law $A(\cdot)$. (For motivations and related discussions, see $[1,2]$.)

A characterization of the stability behavior of $\mathcal{A}$ relies on the sign of the (largest) Lyapunov exponent associated with $\mathcal{A}$, which is defined as

$\rho(\mathcal{A})=\sup \left(\limsup _{t \rightarrow+\infty} \frac{1}{t} \log \|x(t)\|\right)$,

where the sup is taken over the set of solutions of $(1)$ with $x(0) \neq 0$ and $A(\cdot)$ an arbitrary switching law. The Lyapunov exponent is a "measure" of the asymptotic stability of (1). Indeed, the system is (uniformly) exponentially stable if and only if $\rho(\mathcal{A})<0$. This

\footnotetext{
th This work was supported by the French ANR grant ArHyCo, Program ARPEGE, contract number ANR-2008 SEGI 004 01-30011459, by the ANR grant GCM, Program Blanc-CSD, contract number NT09-504490, and by the DIGITEO grant CONGEO.

* Corresponding author.

E-mail addresses: yacine.chitour@lss.supelec.fr (Y. Chitour), paolo.mason@lss.supelec.fr (P. Mason), Mario.Sigalotti@inria.fr (M. Sigalotti).
}

means that there exist $C_{1}, C_{2}>0$ such that, for every trajectory of (1) with $A(\cdot)$ an arbitrary switching law, one has

$\|x(t)\| \leq C_{1} \exp \left(-C_{2} t\right)\|x(0)\|, \quad t \geq 0$.

On the other hand, (1) admits trajectories going to infinity exponentially fast if and only if $\rho(\mathcal{A})>0$. When $\rho(\mathcal{A})=0$, two situations may occur: (i) all trajectories of (1) starting from a bounded set remain uniformly bounded and there exist trajectories staying away from the origin, in which case (1) is said to be marginally stable; (ii) (1) admits a trajectory going to infinity and the system is said to be marginally unstable.

The role of the Lyapunov exponent is analogous to that of the joint spectral radius (or, equivalently, the generalized spectral radius) for discrete-time linear switched systems. The properties of the latter have been studied extensively in recent years (see for instance [3-6]). In particular, for discrete-time linear switched systems, several results have been obtained in the case in which the spectral radius is equal to one under particular assumptions (see for instance [7] and the references therein). This case corresponds to the situation $\rho(\mathcal{A})=0$ for continuous-time systems of the form (1).

The stability properties of continuous-time systems in the case $\rho(\mathcal{A})=0$ have not attracted much attention in the community up to now. Some results, relating marginal stability of (1) to the existence of limit cycles and periodic trajectories can be found in $[8$, 9], while some general observations about marginal stability and instability can be found in [10]. It has to be noted that a qualitative study of the properties of the trajectories in the case $\rho(\mathcal{A})=0$ leads to similar properties for all values of $\rho$, since $\rho\left(\mathcal{A}^{\prime}\right)=0$, where $\mathcal{A}^{\prime}$ is the set $\{A-\rho(\mathcal{A})$ Id $\mid A \in \mathcal{A}\}$ with Id denoting the $n \times n$ identity matrix. 
In this paper, we introduce the concept of resonance, which is rather natural for reducible switched systems with $\rho(\mathcal{A})=0$. Recall that if the switched system is marginally unstable then it must be reducible ([8]), i.e., there exists a linear change of coordinates transforming simultaneously every matrix of $\mathcal{A}$ into an upper block-diagonal matrix (see also Definition 1 below) and the sizes of the blocks do not depend on the particular matrix of $\mathcal{A}$. The diagonal blocks give rise to a finite number of switched systems of lower dimensions $\mathcal{A}_{i}, 1 \leq i \leq r$, each of them being irreducible with nonpositive Lyapunov exponent.

We say that distinct subsystems $\mathcal{A}_{i}$ are in resonance if each of them is marginally stable and they admit trajectories staying away from the origin associated with the same switching law (cf. Definition 6). A characterization of the resonance property in terms of the norms of the evolution operators of the subsystems is given in Proposition 9. We then turn to the relation between resonance and marginal instability. Theorem 10 asserts that if a switched system is marginally unstable, then it admits subsystems in resonance. Clearly, the resonance phenomenon highlighted in Theorem 10 does not guarantee marginal instability (simply consider the case of block-diagonal matrices only). Understanding when marginal instability occurs seems a hard problem since it amounts to understanding how the off-diagonal blocks interconnect the switched systems $\mathcal{A}_{i}, 1 \leq i \leq r$, defined by the diagonal blocks of the elements of $\mathcal{A}$. We nevertheless address this issue in the case where $n \leq 4$ and $\mathcal{A}$ is the convex hull of a set of two matrices $\left\{A^{0}, A^{1}\right\}$. We show that there are no non-trivial examples satisfying the resonance hypothesis for $n=2,3$ and, for $n=4$, we prove that, for almost every choice of the matrices $A^{0}, A^{1}$ satisfying the resonance hypothesis, (1) admits a trajectory going to infinity with linear growth as $t$ tends to infinity.

We next pursue our study of the resonance phenomenon and we characterize an integer $L$, called the resonance degree of $\mathcal{A}$, which measures, in some sense, the complexity of the network of resonance relations between subsystems. In particular, the resonance degree is equal to zero when there is no resonance. We then state and prove our main result, Theorem 19, which provides an upper bound for the norm of the state at time $t$, which is polynomial in $t$ of degree $L$ and which is uniform with respect to the switching law $A(\cdot)$ defined on $[0, t]$. We extend the above study to discrete-time switched systems and obtain results entirely analogous to those dealing with the continuous-time case. In particular we improve an estimate provided in [11], which, to the best of our knowledge, was, up to now, the sharpest estimate on the polynomial behavior of trajectories of marginally unstable discrete-time switched systems.

We conclude the paper with several remarks regarding the worst asymptotic polynomial behavior, and with a comparison with the results and open questions in [7,12]. In particular we estimate both the largest asymptotic growth of single trajectories as the time goes to infinity and the asymptotic growth, as $t$ goes to infinity, of the largest norm of a point attainable at time $t$ from the unit ball. We obtain, rather surprisingly, that, in some cases, the results of the two procedures do not coincide.

\section{Reducibility and definition of resonance}

In this section, we introduce the main notations of the paper as well as the concept of resonance for a reducible switched linear system with zero Lyapunov exponent.

\subsection{Reducibility}

We collect here some well-known facts on reducibility of linear switched systems. If $p$ is a positive integer, we use $\mathcal{M}_{p}(\mathbb{R})$ to denote the real vector space of $p \times p$ matrices with real entries. We fix $n \in \mathbb{N}$ and a compact and convex subset $\mathcal{A}$ of $\mathcal{M}_{n}(\mathbb{R})$. We write $\rho(\mathcal{A})$ for the Lyapunov exponent associated with $\mathcal{A}$, defined as in (2).
Definition 1. We say that

$\{0\}=E_{0} \subsetneq E_{1} \subsetneq \cdots \subsetneq E_{r-1} \subsetneq E_{r}=\mathbb{R}^{n}$,

is an invariant flag for (1) of length $r$ if every $E_{i}$ is a subspace of $\mathbb{R}^{n}$ of dimension $n_{i}$ which is invariant with respect to every matrix $A \in \mathcal{A}$. An invariant flag is said to be maximal if, for every $i=1$, $\ldots, r$, there exists no subspace $V$ such that $E_{i-1} \subsetneq V \subsetneq E_{i}$ and $V$ is invariant with respect to $\mathcal{A}$. Finally an invariant flag is said to be trivial (resp. nontrivial) if $r=1$ (resp. $r>1$ ) and a switched system that admits (resp. does not admit) a nontrivial invariant flag is said to be reducible (resp. irreducible).

The following result relates the stability properties of a reducible switched system to those of lower dimensional irreducible switched systems.

Proposition 2. Given a maximal invariant flag, there exists a vector basis $\left\{v_{1}, \ldots, v_{n}\right\}$ such that, for $i=1, \ldots, r$, one has $E_{i}=\operatorname{span}\left\{v_{1}\right.$, $\ldots, v_{n_{i}}$ \} and such that every matrix $A \in \mathcal{A}$ takes the following block form

$A=\left(\begin{array}{lllll}A_{11} & A_{12} & \ldots & & \\ 0 & A_{22} & A_{23} & \ldots & \\ 0 & 0 & A_{33} & A_{34} & \ldots \\ \vdots & \ddots & \ddots & \ddots & \\ 0 & \cdots & \cdots & 0 & A_{r r}\end{array}\right)$,

where each $A_{i j}$ is an $\left(n_{i}-n_{i-1}\right) \times\left(n_{j}-n_{j-1}\right)$ matrix with real entries. The subsystems of $\mathcal{A}$, defined as the switched systems corresponding to the sets $\mathcal{A}_{i}:=\left\{A_{i i} \mid A \in \mathcal{A}\right\}$ for $i=1, \ldots, r$, are irreducible and satisfy $\rho\left(\mathcal{A}_{i}\right) \leq \rho(\mathcal{A})$ for $1 \leq i \leq r$, with equality holding for at least one index $i$. Moreover, for a fixed switched system $\mathcal{A}$, the length $r$ does not depend on the maximal flag and the subsystems $\mathcal{A}_{i}$ are unique up to reordering, in the sense that, if $\mathcal{A}_{1}, \ldots, \mathcal{A}_{r}$ and $\mathcal{B}_{1}, \ldots, \mathscr{B}_{r^{\prime}}$ are obtained as above, then $r=r^{\prime}$ and there exist a permutation $\sigma$ of $\{1, \ldots, r\}$ and $P_{1}, \ldots, P_{r}$ invertible matrices so that $\mathscr{B}_{i}=$ $P_{i}^{-1} \mathcal{A}_{\sigma(i)} P_{i}$ for $i=1, \ldots, r$.

The first part of the proposition (namely, the reduction into the block form (4)) can be easily proved, as well as the fact that $\rho\left(\mathcal{A}_{i}\right) \leq \rho(\mathcal{A})$ for $1 \leq i \leq r$, thanks to the variation of constants formula and a simple inductive argument. Finally the uniqueness of the subsystems (up to reordering) follows from the Jordan-Hölder theorem for R-modules (see for instance [13, Theorem 13.7]).

In accord with the decomposition described in Proposition 2, we associate with any switching law $A(\cdot)$ in $\mathcal{A}$ and any index $i \in$ $\{1, \ldots, r\}$ a switching law $A_{i i}(\cdot)$ in $\mathcal{A}_{i}$.

From [8], we have the following fundamental result.

Theorem 3. If (1) is irreducible, then there exists a norm $v(\cdot)$ in $\mathbb{R}^{n}$ such that, for every $x_{0} \in \mathbb{R}^{n}$, one has

(a) for every trajectory $x(\cdot)$ of (1) starting from $x_{0}, v(x(t)) \leq v\left(x_{0}\right)$ $\times e^{\rho(\mathcal{A}) t}$, for every $t \geq 0$

(b) there exists a trajectory $x(\cdot)$ of (1) starting from $x_{0}$ satisfying $v(x(t))=v\left(x_{0}\right) e^{\rho(\mathcal{A}) t}$, for every $t \geq 0$.

A norm $v(\cdot)$ satisfying condition $(a)$ is called an extremal norm, while $v(\cdot)$ is called a Barabanov norm if it satisfies both $(a)$ and $(b)$ (see [6]).

Remark 4. An immediate consequence of the previous result is the nontrivial observation that an irreducible switched system (1) with $\rho(\mathcal{A})=0$ is stable and not asymptotically stable. Indeed, the balls with respect to a Barabanov norm are invariant for (1). On the other hand, for every initial condition $x_{0}$, there exists a trajectory of (1) lying on the sphere $v^{-1}\left(v\left(x_{0}\right)\right)$, hence not converging to 0 . 
Remark 5. Let $\rho(\mathcal{A})=0$. Combining Proposition 2 with Theorem 3 and by a simple application of the variation of constants formula, we get that a trajectory can go to infinity at most polynomially. More precisely, there exists $C>0$ such that, for every trajectory of ( 1 ) one has

$\|x(t)\| \leq C\left(1+t^{\hat{r}-1}\right)\|x(0)\|, \quad t \geq 0$,

where $\hat{r}$ is the number of irreducible subsystems associated with $\mathcal{A}$ which are stable and not asymptotically stable. This bound will be sharpened in Section 5 .

\subsection{Resonance}

We now introduce a new concept for the study of linear switched systems, namely, the notion of resonance between irreducible subsystems with zero Lyapunov exponent.

Definition 6. Consider a reducible switched system $\mathcal{A}$ and denote by $\mathcal{A}_{1}, \ldots, \mathcal{A}_{r}$ the subsystems corresponding to a maximal invariant flag, as in Proposition 2. We say that the subsystems $\mathcal{A}_{i_{1}}, \ldots, \mathcal{A}_{i_{s}}$ of $\mathcal{A}$, where $i_{1}, \ldots, i_{s}$ are distinct indices, are in resonance if they satisfy the following conditions,

(a) $\rho\left(\mathcal{A}_{i_{1}}\right)=\cdots=\rho\left(\mathcal{A}_{i_{s}}\right)=0$;

(b) there exists a switching law $A(\cdot)$ in $\mathcal{A}$ with associated switching laws $A_{i_{j} i_{j}}(\cdot)$ in $\mathcal{A}_{i_{j}}$ and corresponding trajectories $\gamma_{i_{j}}(\cdot)$ of $\mathcal{A}_{i_{j}}$ such that, for every $t>0$ and for $j=1, \ldots, s, \gamma_{i_{j}}(t)$ belongs to the sphere $v_{i_{j}}^{-1}(1)$, where $v_{i_{j}}$ is a Barabanov norm associated with $\mathcal{A}_{i_{j}}$.

The concept of resonance does not actually depend on the specific Barabanov norms appearing in condition $(b)$ in the above definition. The latter fact is a consequence of the following lemma.

Lemma 7. We use the notations of Definition 6. Assume that the subsystems $\mathcal{A}_{i_{1}}, \ldots, \mathcal{A}_{i_{s}}$ of $\mathcal{A}$ are in resonance and let $w_{i_{1}}(\cdot), \ldots$, $w_{i_{s}}(\cdot)$ be extremal norms associated with $\mathcal{A}_{i_{1}}, \ldots, \mathcal{A}_{i_{s}}$. Then there exists a switching law $\tilde{A}(\cdot)$ in $\mathcal{A}$ with associated switching laws $\tilde{A}_{i_{j} i_{j}}(\cdot)$ in $\mathcal{A}_{i_{j}}$ and corresponding trajectories $\tilde{\gamma}_{i_{j}}(\cdot)$ of $\mathcal{A}_{i_{j}}$ such that, for every $t>0$ and for $j=1, \ldots, s, \tilde{\gamma}_{i_{j}}(t)$ belongs to the sphere $w_{i_{j}}^{-1}(1)$.

Proof. Let $A(\cdot)$ be as in condition (b) of Definition 6. Consider a sequence $\left(t_{k}\right)_{k \in \mathbb{N}}$ of positive times going to infinity and such that $\gamma_{i_{j}}\left(t_{k}\right)$ converges to some $\bar{\gamma}_{i_{j}}$ for $j=1, \ldots, s$. Since $\mathcal{A}$ is convex and compact the space $L^{\infty}([0,+\infty), \mathcal{A})$ is closed in the weak-* topology and, by the Banach-Alaoglu theorem, there exists a weak-* limit of $A\left(t_{k}+\cdot\right)$ in $L^{\infty}([0,+\infty)$, A $)$, i.e., up to a subsequence, $A\left(t_{k}+\cdot\right) \stackrel{w^{*}}{\rightarrow} A^{*}(\cdot)$ in $L^{\infty}([0,+\infty), \mathcal{A})$. In particular, for $j=1, \ldots, s, A_{i_{j}}\left(t_{k}+\cdot\right) \stackrel{w^{*}}{\rightarrow} A_{i_{j}}^{*}(\cdot)$ in $L^{\infty}\left([0,+\infty), \mathcal{A}_{i_{j}}\right)$ and (see for instance [14]) $\gamma_{i_{j}}\left(t_{k}+\cdot\right)$ converges uniformly on compact timeintervals to a solution $\gamma_{i_{j}}^{*}(\cdot)$ of $\mathcal{A}_{i_{j}}$ with $\gamma_{i_{j}}^{*}(0)=\bar{\gamma}_{i_{j}}$. Since $w_{i_{j}}$ is extremal, $\lim _{\tau \rightarrow \infty} w_{i_{j}}\left(\gamma_{i_{j}}(\tau)\right)$ exists. Hence,

$$
\begin{aligned}
w_{i_{j}}\left(\gamma_{i_{j}}^{*}(t)\right) & =\lim _{k \rightarrow \infty} w_{i_{j}}\left(\gamma_{i_{j}}\left(t_{k}+t\right)\right)=\lim _{k \rightarrow \infty} w_{i_{j}}\left(\gamma_{i_{j}}\left(t_{k}\right)\right) \\
& =w_{i_{j}}\left(\bar{\gamma}_{i_{j}}\right)=w_{i_{j}}\left(\gamma_{i_{j}}^{*}(0)\right) .
\end{aligned}
$$

The thesis is then satisfied with $\tilde{A}(\cdot)=A^{*}(\cdot)$ and $\tilde{\gamma}_{i_{j}}(\cdot)=$ $\gamma_{i_{j}}^{*}(\cdot) / w_{i_{j}}\left(\gamma_{i_{j}}^{*}(0)\right)$.

Example 8 (Resonance for Low-Dimensional Systems). Since onedimensional systems with zero Lyapunov exponent are trivial, the first interesting resonances appear between pairs of twodimensional (irreducible) systems.
Stability behavior of planar linear switched systems $\mathcal{A}=\operatorname{co}\left\{A^{0}\right.$, $A^{1}$ \} (that is, for which $\mathcal{A}$ is the convex hull of two matrices $A^{0}$ and $A^{1}$ ) has been characterized in a series of papers, first in the case where the rank of $A^{1}-A^{0}$ is equal to one and then in the general case (see [15-19]). It is well known, in particular, that $\rho(\mathcal{A})=0$ with all the matrices of $\mathcal{A}$ being Hurwitz if and only if $\mathcal{A}$ admits a closed worst-case trajectory, which is a trajectory whose support is the Barabanov sphere (which is unique in this case). The worst-case trajectory corresponds to a periodic switching law $A(\cdot)$ obtained by concatenating a time-interval of length $t_{0}>0$ on which $A(t)=A^{0}$ with a second one of length $t_{1}>0$ on which $A(t)=A^{1}$ and then extending by periodicity. The times $t_{0}, t_{1}$ are called switching times. The period of the closed worst-case trajectory is equal to $2 t_{0}+2 t_{1}$, i.e., the worst-case trajectory is the concatenation of four bang arcs, whose supports constitute the Barabanov sphere. Then the worstcase trajectory is the only trajectory staying on the Barabanov sphere and two planar switched systems as above are in resonance if and only if the corresponding switching times coincide.

A similar characterization can be obtained for resonances between a two-dimensional and a three-dimensional system, provided that the latter is defined by the convex hull of two matrices whose difference has rank equal to one. This case has been studied in [20] (see also [9]) where it is proved that the system has a unique periodic trajectory on a Barabanov sphere. Such periodic trajectory, moreover, is symmetric with respect to the origin and is made by the concatenation of four bang arcs. Although in this case the periodic trajectory is not the only one lying on the Barabanov sphere, it is the only one staying in the $\omega$-limit set of trajectories lying on it. It can be easily proved that if a switching law $A(\cdot)$ as in point $(b)$ of the definition of resonance exists, then, possibly replacing $A(\cdot)$ by another switching law, we can impose the additional requirement that all trajectories $\gamma_{i_{j}}$ stay in the $\omega$-limit set of admissible trajectories lying on $v_{i_{j}}^{-1}(1)$. Hence, as in the case of pairs of planar switched systems, resonance holds if and only if the switching times of the two-dimensional system coincide with those of the periodic trajectory of the threedimensional system.

We now provide an equivalent definition of the concept of resonance in terms of evolution operators associated with the subsystems of $\mathcal{A}$. We recall that the evolution operator corresponding to a linear time-varying system $\dot{x}(t)=A(t) x(t)$ is the matrix-valued function $R(\cdot, \cdot)$ satisfying $\frac{d}{d t} R(t, s)=A(t) R(t, s)$ and $R(s, s)=\mathrm{Id}$. We also recall that with any norm $v(\cdot)$ on $\mathbb{R}^{n}$ it is associated the matrix norm

$\|M\|_{v}:=\max _{\substack{z \in \mathbb{R}^{n} \\ v(z)=1}} v(M z)$.

Notice that $\|\cdot\|_{v}$ is sub-multiplicative, i.e., for every pair of matrices $M_{1}, M_{2}$ in $\mathcal{M}_{n}(\mathbb{R})$, one has $\left\|M_{1} M_{2}\right\|_{v} \leq\left\|M_{1}\right\|_{v}\left\|M_{2}\right\|_{v}$. By $\|\cdot\|$ we denote, as usual, the matrix norm corresponding to the Euclidean norm.

Proposition 9. Fix a linear switched system $\mathcal{A}$ with $\rho(\mathcal{A})=0$ and with subsystems $\mathcal{A}_{1}, \ldots, \mathcal{A}_{r}$. Then, for every $\bar{C}>0$ small enough there exist $\bar{T}>0$ (depending in general on $\bar{C}$ ) such that the subsystems corresponding to a subset I of $\{1, \ldots, r\}$ are in resonance if and only if there exists a switching law $A(\cdot)$ such that

$I \subset\left\{i \in\{1, \ldots, r\} \mid\left\|R_{i}(\bar{T}, 0)\right\|>\bar{C}\right\}$,

where $R_{i}$ is the evolution operator associated with $A_{i i}(\cdot)$.

Proof. Without loss of generality $\rho\left(\mathcal{A}_{i}\right)=0$ for every $i=1, \ldots, r$. Denote by $v_{i}$ a Barabanov norm associated with $\mathcal{A}_{i}, i=1, \ldots, r$.

Notice that, by equivalence of all matrix norms on a finitedimensional matrix space, we can equivalently replace (6) by

$I \subset\left\{i \in\{1, \ldots, r\} \mid\left\|R_{i}(\bar{T}, 0)\right\|_{i}>\bar{C}\right\}$, 
where $\|\cdot\|_{i}$ denotes the matrix norm $\|\cdot\|_{v_{i}}$ on $\mathcal{M}_{n_{i}-n_{i-1}}(\mathbb{R})$ associated with $v_{i}$.

If the subsystems $\mathscr{A}_{i}, i \in I$, are in resonance, then (7) is satisfied for every $\bar{T}>0$ and $\bar{C} \in(0,1)$, taking as $A(\cdot)$ the switching law appearing in the definition of resonance.

We then focus on the "if" part of the statement. We first prove that there exist $\hat{T}>0$ and $\hat{C} \in(0,1)$ such that, for every switching law $A(\cdot)$, the subsystems of $\mathcal{A}$ corresponding to the indices in

$\hat{I}(A(\cdot))=\left\{i \in\{1, \ldots, r\} \mid\left\|R_{i}(\hat{T}, 0)\right\|_{i}>\hat{C}\right\}$

are in resonance.

By contradiction, assume there exist a sequence $T_{k}$ converging to infinity, a sequence $C_{k} \in(0,1)$ converging to 1 and a switching law $A^{(k)}(\cdot)$ (whose corresponding evolution operator is denoted by $\left.R^{(k)}\right)$ such that the subsystems corresponding to the indices in

$I_{k}\left(A^{(k)}(\cdot)\right)=\left\{i \in\{1, \ldots, r\} \mid\left\|R_{i}^{(k)}\left(T_{k}, 0\right)\right\|_{i}>C_{k}\right\}$,

are not in resonance. Up to extracting a subsequence, we can assume that the set $I:=I_{k}\left(A^{(k)}(\cdot)\right)$ does not depend on $k$. For every $i \in I$, let $x_{i}^{(k)}(\cdot)$ be a solution of $\dot{x}_{i}^{(k)}(t)=A_{i i}^{(k)}(t) x_{i}^{(k)}(t)$ such that $v_{i}\left(x_{i}^{(k)}(0)\right)=1$ and $v_{i}\left(x_{i}^{(k)}\left(T_{k}\right)\right)>C_{k}$. As recalled in the proof of Lemma 7, up to a subsequence, $A^{(k)}(\cdot) \stackrel{w^{*}}{\rightarrow} A^{*}(\cdot)$ in $L^{\infty}([0,+\infty), \mathcal{A})$, and $x_{i}^{(k)}(\cdot) \stackrel{L_{\mathrm{loc}}^{\infty}([0, \infty))}{\longrightarrow} x_{i}^{*}(\cdot)$ for $i \in I$, where $x_{i}^{*}(\cdot)$ is a solution of the switched system $\mathcal{A}_{i}$ corresponding to $A^{*}(\cdot)$. In particular $v_{i}\left(x_{i}^{*}(t)\right)=1$ for $t>0$ and $i \in I$, contradicting the hypothesis that the subsystems $\mathcal{A}_{i}, i \in I$, are not in resonance. The existence of $\hat{T}$ and $\hat{C}$ as above is proved.

Let now $\bar{C} \in(0,1)$. We have to prove that there exists $\bar{T}>0$ such that for every switching law $A(\cdot)$ the subsystems corresponding to the indices in $\left\{i \in\{1, \ldots, r\} \mid\left\|R_{i}(\bar{T}, 0)\right\|_{i}>\bar{C}\right\}$ are in resonance. Let $m \in \mathbb{N}$ be such that $\hat{C}^{m} \leq \bar{C}$ and $M \leq 2^{r}$ be the number of subsets of $\{1, \ldots, r\}$ whose corresponding subsystems are in resonance. Set $\bar{T}=(M(m-1)+1) \hat{T}$. The choice of $\bar{T}$ is such that, for every switching law $A(\cdot)$, the map associating with $j \in\{0, \ldots, M(m-1)\}$ the set $\hat{I}(A(\cdot+j \hat{T}))$ takes at least $m$ times the same value $F \subset\{1, \ldots, r\}$. For any index $i \in\{1, \ldots, r\} \backslash F$,

$\left\|R_{i}(\bar{T}, 0)\right\|_{i} \leq \Pi_{j=0}^{M(m-1)}\left\|R_{i}((j+1) \hat{T}, j \hat{T})\right\|_{i} \leq \hat{C}^{m} \leq \bar{C}$.

Hence, $\left\{i \in\{1, \ldots, r\} \mid\left\|R_{i}(\bar{T}, 0)\right\|_{i}>\bar{C}\right\}$ is a subset of $F$, which implies that the subsystems corresponding to its indices are in resonance.

\section{Resonance as a necessary condition for marginal instability}

We can now state, in terms of the resonance notion introduced in the previous section, the following necessary condition for marginal instability.

Theorem 10. Let $\mathcal{A}$ be a convex compact subset of $\mathcal{M}_{n}(\mathbb{R})$. Assume that the linear switched system associated with $\mathcal{A}$ is marginally unstable. Then $\mathcal{A}$ is reducible and, for any maximal invariant flag, it admits two subsystems $\mathcal{A}_{i_{1}}, \mathcal{A}_{i_{2}}$ in resonance.

Proof. Let us prove that, if there are no subsystems of $\mathcal{A}$ in resonance, then the system is stable. Fix a maximal invariant flag such that all the matrices of $\mathcal{A}$ are of the form (4) and let $x=\left(x_{1}, \ldots, x_{r}\right)$ where $x_{i} \in \mathbb{R}^{n_{i}-n_{i-1}}$ for $i=1, \ldots, r$. Consider a switching law $A(\cdot) \in \mathcal{A}$ and let $R_{i}(t, \tau)$, for $\tau, t \in \mathbb{R}$, be the evolution operator of the time-varying linear system $\dot{z}_{i}=A_{i i} z_{i}, z_{i} \in \mathbb{R}^{n_{i}-n_{i-1}}$, i.e., $z_{i}(t)=R_{i}(t, \tau) z_{i}(\tau)$.

In particular, we have $x_{r}(t)=R_{r}(t, 0) x_{r}(0)$ and, since

$$
\begin{aligned}
\dot{x}_{r-1}(t) & =A_{r-1, r-1}(t) x_{r-1}(t)+A_{r-1, r}(t) x_{r}(t) \\
& =A_{r-1, r-1}(t) x_{r-1}(t)+A_{r-1, r}(t) R_{r}(t, 0) x_{r}(0),
\end{aligned}
$$

by the variation of constants formula, we get

$$
\begin{aligned}
x_{r-1}(t)= & R_{r-1}(t, 0) x_{r-1}(0) \\
& +\int_{0}^{t} R_{r-1}(t, \tau) A_{r-1, r}(\tau) R_{r}(\tau, 0) x_{r}(0) .
\end{aligned}
$$

Repeating recursively the previous computations, we get

$x_{i}(t)=R_{i}(t, 0) x_{i}(0)+\sum_{s=1}^{r-i} \sum_{i<i_{1}<\cdots<i_{s} \leq r} I\left(t, i, i_{1}, \ldots, i_{s}\right) x_{i_{s}}(0)$,

where the integral $I\left(t, i, i_{1}, \ldots, i_{s}\right)$ is defined as

$$
\begin{aligned}
& \int_{t \geq \tau_{1} \geq \cdots \geq \tau_{s} \geq 0} R_{i}\left(t, \tau_{1}\right) A_{i, i_{1}}\left(\tau_{1}\right) \\
& \quad \times R_{i_{1}}\left(\tau_{1}, \tau_{2}\right) \cdots A_{i_{s-1}, i_{s}}\left(\tau_{s}\right) R_{i_{s}}\left(\tau_{s}, 0\right) d \tau_{1} \cdots d \tau_{s} .
\end{aligned}
$$

We prove the stability of $\mathscr{A}$ using the inequality

$$
\begin{aligned}
\left\|x_{i}(t)\right\| \leq & \left(\left\|R_{i}(t, 0)\right\|\right. \\
& \left.+\sum_{s=1}^{r-i} \sum_{i<i_{1}<\cdots<i_{s} \leq r}\left\|I\left(t, i, i_{1}, \ldots, i_{s}\right)\right\|\right)\|x(0)\|,
\end{aligned}
$$

and by estimating each $\left\|I\left(t, i, i_{1}, \ldots, i_{s}\right)\right\|$. Denote by $\|\cdot\|_{i}, 1 \leq$ $i \leq r$, the matrix norm $\|\cdot\|_{v_{i}}$ corresponding to a Barabanov norm $v_{i}$ associated with $\mathscr{A}_{i}$. Since two norms defined on a finite dimensional vector space are equivalent, there exists $K_{i}>0$ such that $\|M\| \leq K_{i}\|M\|_{i}$ for $i=1, \ldots, k$. Finally, by definition, we have $\left\|R_{i}\left(\tau_{1}, \tau_{2}\right)\right\|_{i} \leq e^{\rho\left(\mathcal{A}_{i}\right)\left(\tau_{1}-\tau_{2}\right)}$ for every choice of the switching law and $\tau_{2}<\tau_{1}$. Since $\mathcal{A}$ is compact, there exists $K>0$ independent of the switching law such the following holds true,

$$
\begin{aligned}
& \| \int_{t \geq \tau_{1} \geq \cdots \geq \tau_{s} \geq 0} R_{i}\left(t, \tau_{1}\right) A_{i, i_{1}}\left(\tau_{1}\right) \\
& \quad \times R_{i_{1}}\left(\tau_{1}, \tau_{2}\right) \cdots A_{i_{s-1}, i_{s}}\left(\tau_{s}\right) R_{i_{s}}\left(\tau_{s}, 0\right) d \tau_{1} \cdots d \tau_{s} \| \\
& \leq \int_{t \geq \tau_{1} \geq \cdots \geq \tau_{s} \geq 0} \| R_{i}\left(t, \tau_{1}\right) A_{i, i_{1}}\left(\tau_{1}\right) \\
& \quad \times R_{i_{1}}\left(\tau_{1}, \tau_{2}\right) \cdots A_{i_{s-1}, i_{s}}\left(\tau_{s}\right) R_{i_{s}}\left(\tau_{s}, 0\right) \| d \tau_{1} \cdots d \tau_{s} \\
& \leq K \int_{t \geq \tau_{1} \geq \cdots \geq \tau_{s} \geq 0}\left\|R_{i}\left(t, \tau_{1}\right)\right\|_{i}\left\|R_{i_{1}}\left(\tau_{1}, \tau_{2}\right)\right\|_{i_{1}} \cdots \\
& \quad \times\left\|R_{i_{s}}\left(\tau_{s}, 0\right)\right\|_{i_{s}} d \tau_{1} \cdots d \tau_{s} .
\end{aligned}
$$

Given $T>0$ and using [.] to denote the integer part of a real number, there exists $K^{\prime} \geq 1$ depending on $T$ but not on $t$ nor on the switching law, such that

$$
\begin{aligned}
& \int_{t \geq \tau_{1} \geq \cdots \geq \tau_{s} \geq 0}\left\|R_{i}\left(t, \tau_{1}\right)\right\|_{i}\left\|R_{i_{1}}\left(\tau_{1}, \tau_{2}\right)\right\|_{i_{1}} \cdots \\
& \times\left\|R_{i_{s}}\left(\tau_{s}, 0\right)\right\|_{i_{s}} d \tau_{1} \cdots d \tau_{s} \\
& \leq K^{\prime} \int_{\left[\frac{t}{T}\right] T+T \geq \tau_{1} \geq \cdots \geq \tau_{s} \geq 0}\left\|R_{i}\left(\left[\frac{t}{T}\right] T+T,\left[\frac{\tau_{1}}{T}\right] T\right)\right\|_{i} \\
& \times\left\|R_{i_{1}}\left(\left[\frac{\tau_{1}}{T}\right] T,\left[\frac{\tau_{2}}{T}\right] T\right)\right\|_{i_{1}} \cdots \\
& \times\left\|R_{i_{s}}\left(\left[\frac{\tau_{s}}{T}\right] T, 0\right)\right\|_{i_{s}} d \tau_{1} \cdots d \tau_{s} \\
& \leq K^{\prime} T^{s} \sum_{0 \leq m_{s} \leq \cdots \leq m_{0}=\left[\frac{t}{T}\right]+1}\left\|R_{i}\left(m_{0} T, m_{1} T\right)\right\|_{i} \\
& \times\left\|R_{i_{1}}\left(m_{1} T, m_{2} T\right)\right\|_{i_{1}} \cdots\left\|R_{i_{s}}\left(m_{s} T, 0\right)\right\|_{i_{s}}
\end{aligned}
$$




$$
\begin{aligned}
\leq & K^{\prime} T^{s} \sum_{0 \leq m_{s} \leq \cdots \leq m_{0}} \prod_{j=1}^{m_{s}}\left\|R_{i_{s}}(j T,(j-1) T)\right\|_{i_{s}} \cdots \\
& \times \prod_{j=m_{2}+1}^{m_{1}}\left\|R_{i_{1}}(j T,(j-1) T)\right\|_{i_{1}} \prod_{j=m_{1}+1}^{m_{0}}\left\|R_{i}(j T,(j-1) T)\right\|_{i} .
\end{aligned}
$$

We are now left to prove that the previous sum is uniformly bounded with respect to the choice of the switching law and independently of $t$, at least when $T$ is large enough. According to Proposition 9, since $\mathcal{A}$ does not have subsystems in resonance, there exists $C \in(0,1)$ and $T>0$ such that, for every pair of distinct indices $(i, j)$ with $1 \leq i, j \leq r$ and for every switching law,

$\left\|R_{i}(T, 0)\right\|_{i}\left\|R_{j}(T, 0)\right\|_{j} \leq C$.

The proof of the theorem is then concluded, in view of (9) and (10), by applying to the sum of products in (11) the combinatorial result Lemma 28 stated and proved in the appendix.

Remark 11. The combined results of Proposition 2 and Theorem 10 are in the same spirit as Lemma 1 in [10], where the author states that a marginally unstable system admits a (possibly non-maximal) proper invariant flag of length two identifying two subsystems $\mathscr{A}_{1}, \mathcal{A}_{2}$ with $\rho\left(\mathcal{A}_{1}\right)=\rho\left(\mathcal{A}_{2}\right)=0$. It should be noticed that the conclusion in [10, Lemma 1] states in addition that both $\mathcal{A}_{1}$ and $\mathcal{A}_{2}$ can be taken marginally stable. However the latter statement is not true in general. Indeed, consider the switched system given by

$\mathcal{A}=\left\{\left(\begin{array}{lll}0 & 1 & 0 \\ 0 & 0 & 1 \\ 0 & 0 & 0\end{array}\right)\right\}$.

The only proper invariant subspaces of $\mathcal{A}$ are $\mathbb{R} \times\{(0,0)\}$ and $\mathbb{R}^{2} \times\{(0)\}$. Hence, one of the two subsystems associated with $\mathcal{A}$ is marginally unstable.

\section{Sufficient conditions for marginal instability: the low- dimensional cases}

A natural question arising from the results of the previous section is whether, or under which additional conditions, a switched system with $\rho(\mathcal{A})=0$ and which admits subsystems in resonance is marginally unstable. A simple observation is that if $\rho(\mathcal{A})=0$ and if we assume that there exists a vector basis such that each matrix of $\mathcal{A}$ can be put in the block form (4) with $A_{i j}=0$ for $i<j$ then the switched system (1) is stable, independently of the existence or non-existence of subsystems in resonance. Indeed in this case, setting $x=\left(x_{1}, \ldots, x_{r}\right)$ where $x_{i} \in \mathbb{R}^{n_{i}-n_{i+1}}$ for $i=1, \ldots, r$, the components $x_{i}$ of a trajectory of (1) vary independently one from each other and the stability of the overall system is therefore guaranteed by the fact that $\rho\left(\mathcal{A}_{i}\right) \leq 0$ (see Proposition 2). The role of the interaction terms $A_{i j}$ is therefore fundamental to possibly show the existence of trajectories going to infinity. In the general case, a complete analysis of the contribution of these interaction terms is definitely a hard issue to address. Therefore we will limit ourselves to low-dimensional systems of the form $\mathcal{A}=\operatorname{co}\left\{A^{0}, A^{1}\right\}$.

To state some of our subsequent results, we need the following definition.

Definition 12. A marginally unstable switched system associated with a convex compact subset $\mathcal{A}$ of $\mathcal{M}_{n}(\mathbb{R})$ is said to be polynomially unstable of degree $l$ if there exists a positive integer $l$ and constants $C_{1}, C_{2}>0$ such that every solution $x(\cdot)$ of (1) satisfies $\|x(t)\| \leq$ $C_{1}\left(1+t^{l}\right)\|x(0)\|$ and there exists a solution $\bar{x}(\cdot)$ of (1) satisfying $\|\bar{x}(t)\| \geq C_{2} t^{l}\|\bar{x}(0)\|$ for every $t>0$.
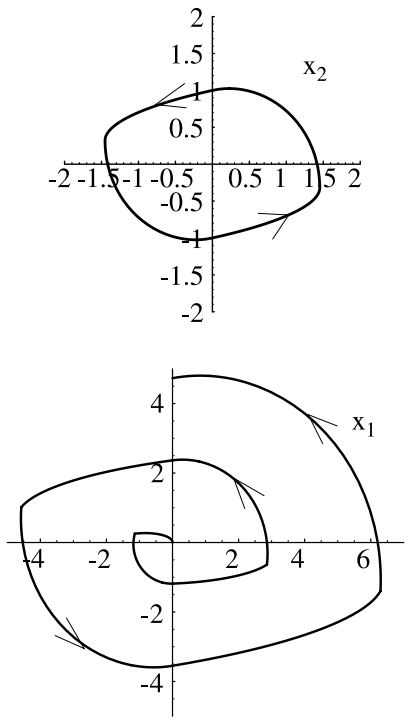

Fig. 1. Trajectory of a polynomially unstable switched system as in Example 13.

Let $\mathcal{A}=\operatorname{co}\left\{A^{0}, A^{1}\right\} \subset \mathcal{M}_{n}(\mathbb{R})$ be a marginally unstable system. We consider the particular case in which all the matrices $u A^{0}+$ $(1-u) A^{1}$, where $u \in[0,1]$, are Hurwitz.

As a consequence of Theorem 10, it turns out immediately that $n \geq 4$, since otherwise one of the two subsystems obtained by applying Theorem 10 would be of dimension one and its maximal Lyapunov exponent would be equal to zero, leading to one of the two matrices $A^{0}, A^{1}$ having 0 as eigenvalue.

Let us fix $n=4$. Marginal instability can only occur if the following holds true: there is a maximal invariant flag (3) with $k=2$, $n_{1}=2$ and, for the associated subsystems, $\rho\left(\mathscr{A}_{1}\right)=\rho\left(\mathscr{A}_{2}\right)=0$. In particular, we can write the matrices $A^{0}, A^{1}$ in the block form

$A^{0}=\left(\begin{array}{cc}A_{11}^{0} & A_{12}^{0} \\ 0 & A_{22}^{0}\end{array}\right), \quad A^{1}=\left(\begin{array}{cc}A_{11}^{1} & A_{12}^{1} \\ 0 & A_{22}^{1}\end{array}\right)$,

where all the blocks are $2 \times 2$. As recalled in Example 8 the planar switched systems $\mathcal{A}_{*}=\operatorname{co}\left\{A_{*}^{0}, A_{*}^{1}\right\}$, with $\rho\left(\mathcal{A}_{*}\right)=0$ and such that all the matrices of $\mathcal{A}_{*}$ are Hurwitz, are those admitting a closed bang-bang worst-case trajectory characterized by the switching times $t_{0}, t_{1}>0$. Let us denote by $T$ the half-period $t_{0}+t_{1}$ of the worst-case trajectory.

It is now easy to build an example of polynomially unstable switched system with matrices in the block form (12).

Example 13. Assume that $A^{0}, A^{1}$ in (12) are such that $A_{11}^{0}=A_{22}^{0}=$ $A_{*}^{0}, A_{11}^{1}=A_{22}^{1}=A_{*}^{1}$ and $A_{12}^{0}=A_{12}^{1}=$ Id, where $\mathcal{A}_{*}=\operatorname{co}\left\{A_{*}^{0}, A_{*}^{1}\right\}$ satisfies the properties detailed above. If the evolution operator $R_{*}(t, 0)$ corresponds to a worst-case switching strategy for $\mathcal{A}_{*}$ one can immediately verify, with the variation of constants formula, that

$$
\begin{aligned}
& x_{2}(t)=R_{*}(t, 0) x_{2}(0), \\
& x_{1}(t)=R_{*}(t, 0) x_{1}(0)+t R_{*}(t, 0) x_{2}(0),
\end{aligned}
$$

so that the system is polynomially unstable of degree 1 . An explicit numerical example can be obtained by considering the matrices

$A_{*}^{0}=\left(\begin{array}{cc}-1 & -\alpha \\ \alpha & -1\end{array}\right), \quad A_{*}^{1}=\left(\begin{array}{cc}-1 & -\alpha \\ 1 / \alpha & -1\end{array}\right)$.

For a value $\alpha \sim 4.5047$, one has $\rho\left(\mathcal{A}_{*}\right)=0$ and Fig. 1 depicts a particular trajectory for such a value. 
In the following we consider closed worst-case trajectories of the form $t \mapsto R_{*}(t, 0) \bar{x}_{1}$, for some $\bar{x}_{1} \neq 0$, where the evolution operator $R_{*}(t, 0)$ corresponds to the switching law

$A_{*}(t)= \begin{cases}A_{*}^{0} & \text { if } t \in\left[k T, k T+t_{0}\right), k \in \mathbb{N}, \\ A_{*}^{1} & \text { if } t \in\left[k T+t_{0},(k+1) T\right), k \in \mathbb{N} .\end{cases}$

It turns out that $\bar{x}_{1}$ is an eigenvector of the matrix $R_{*}(T, 0)$ corresponding to the eigenvalue -1 , while the modulus of the second eigenvalue is strictly smaller than one.

For switched systems $\mathcal{A}=\operatorname{co}\left(A^{0}, A^{1}\right)$, where $A^{0}, A^{1}$ are given by (12), Theorem 10 admits the following converse result (note that condition (C1) below is equivalent to requiring that $\mathcal{A}_{1}$ and $\mathcal{A}_{2}$ are in resonance).

Theorem 14. Let $A^{0}, A^{1}$ be two $4 \times 4$ Hurwitz matrices in the block form (12). Assume that

(C1) the switched systems $\mathcal{A}_{1}=\operatorname{co}\left\{A_{11}^{0}, A_{11}^{1}\right\}$ and $\mathcal{A}_{2}=\operatorname{co}\left\{A_{22}^{0}\right.$, $\left.A_{22}^{1}\right\}$ admit closed worst-case trajectories with the same switching times $t_{0}, t_{1}$,

(C2) the condition

$\int_{0}^{T} \Pi_{x_{1}^{1}}\left(R_{1}(T, \tau) A_{12}(\tau) R_{2}(\tau, 0) x_{1}^{2}\right) d \tau \neq 0$

is satisfied. Here $A(\cdot)$ is the signal giving rise to the worst-case trajectory (cf. (13)), $A_{12}(\cdot)$ denotes its top right $2 \times 2$ block, and $R_{k}(\cdot, \cdot), k=1,2$, are the evolution operators associated with its subsystems. Moreover, $T=t_{0}+t_{1}, x_{1}^{k}, x_{2}^{k}$ are eigenvectors of $R_{k}(T, 0)$, for $k=1,2$, and $\Pi_{x_{1}^{1}}(x)$ is the component of the vector $x$ along the direction $x_{1}^{1}$ with respect to the basis $\left\{x_{1}^{1}, x_{2}^{1}\right\}$.

Then (1) is polynomially unstable of degree one. Moreover assume that $\mathcal{A}_{1}$ and $\mathcal{A}_{2}$ satisfy Condition (C1). Then, (C2) is satisfied for every pair of matrices $\left(A_{12}^{0}, A_{12}^{1}\right)$ in the complement of an hyperplane of $\mathcal{M}_{2}(\mathbb{R}) \times \mathcal{M}_{2}(\mathbb{R})$.

Proof. Let us consider the trajectory of (1) starting at $\left(0, x_{1}^{2}\right)^{T}$ and corresponding to the worst-case switching strategy for $\mathcal{A}_{1}, \mathcal{A}_{2}$,

$x_{2}(t)=R_{2}(t, 0) x_{1}^{2}$

$x_{1}(t)=\int_{0}^{t} R_{1}(t, \tau) A_{12}(\tau) R_{2}(\tau, 0) x_{1}^{2} d \tau$.

We first prove that the system is polynomially unstable under the hypotheses of the theorem. Fix $\tau \in[0, t]$ and consider the integer part $q(\tau)=\left[\frac{\tau}{T}\right]$ and the remainder $r(\tau)=\tau-T\left[\frac{\tau}{T}\right]$. Notice that

(I) the matrix $A_{12}(\cdot)$ only depends on $r(\tau)$, i.e.,

$A_{12}(\tau)=A_{12}(r(\tau))$,

(II) $R_{1}\left(\tau_{1}, \tau_{2}\right)=R_{1}\left(\tau_{1}+T, \tau_{2}+T\right)$ and $R_{2}\left(\tau_{1}, \tau_{2}\right)=R_{2}\left(\tau_{1}+T, \tau_{2}+\right.$ $T$ ) for every $\tau_{1}, \tau_{2}>0$, since the period of the switching law is $T$,

(III) for all $0 \leq \tau \leq m T, m \in \mathbb{N}$, we can write

$$
\begin{aligned}
& R_{1}(m T, \tau)=R_{1}(m T,(q(\tau)+1) T) R_{1}((q(\tau)+1) T, \tau), \\
& R_{2}(\tau, 0)=R_{2}(\tau, q(\tau) T) R_{2}(T, 0)^{q(\tau)},
\end{aligned}
$$

(IV) by definition of $\Pi_{x_{1}^{1}}$, if $v=v_{1} x_{1}^{1}+v_{2} x_{2}^{1} \in \mathbb{R}^{2}$, we have

$$
\begin{aligned}
\Pi_{x_{1}^{1}}\left(R_{1}(T, 0) v\right)= & v_{1} \Pi_{x_{1}^{1}}\left(R_{1}(T, 0) x_{1}^{1}\right) \\
& +v_{2} \Pi_{x_{1}^{1}}\left(R_{1}(T, 0) x_{2}^{1}\right)=-v_{1}=-\Pi_{x_{1}^{1}}(v) .
\end{aligned}
$$

Combining these facts we easily get

$$
\begin{aligned}
& \Pi_{x_{1}^{1}}\left(\int_{0}^{m T} R_{1}(m T, \tau) A_{12}(\tau) R_{2}(\tau, 0) x_{1}^{2} d \tau\right) \\
& \quad=(-1)^{m+1} m \int_{0}^{T} \Pi_{x_{1}^{1}}\left(R_{1}(T, \tau) A_{12}(\tau) R_{2}(\tau, 0) x_{1}^{2}\right) d \tau .
\end{aligned}
$$

Then, under Condition (C2), $\left|\Pi_{x_{1}^{1}}\left(x_{1}(m T)\right)\right| \geq C_{1} m$, so that $\|x(m T)\|$ $\geq C_{2} m$ and, by the continuity of the evolution operator of (1), we easily get $\|x(t)\| \geq C_{3} t$, for suitable strictly positive constants $C_{1}, C_{2}, C_{3}$. The proof of the first part of the theorem is complete. We are left to prove that Condition (C2) is satisfied in the complement of an hyperplane of $\mathcal{M}_{2}(\mathbb{R}) \times \mathcal{M}_{2}(\mathbb{R})$ (in particular, that $(\mathrm{C} 2)$ is satisfied generically with respect to the choice of the matrices $\left.\left(A_{12}^{0}, A_{12}^{1}\right)\right)$. This is a straightforward consequence of the following lemma.

Lemma 15. With the notations above, let $y$ be a nonzero vector in $\mathbb{R}^{2}$ orthogonal to $x_{2}^{1}$. Then, the linear map

$\Psi: \mathcal{M}_{2}(\mathbb{R}) \times \mathcal{M}_{2}(\mathbb{R}) \rightarrow \mathbb{R}$
$\left(A_{12}^{0}, A_{12}^{1}\right) \mapsto \int_{0}^{T} y^{T} R_{1}(T, \tau) A_{12}(\tau) R_{2}(\tau, 0) x_{1}^{2} d \tau$

is onto.

Proof. Worst-case trajectories are such that, as $\tau$ varies in $\left[0, t_{0}\right]$, the vector $R_{2}(\tau, 0) x_{1}^{2}$ lies in one of the four positive cones defined by the lines $\mathbb{R} x_{1}^{2}$ and $\mathbb{R} x_{2}^{2}$. Each of these cones is strictly contained in a half-plane. In particular, there exists $w \in \mathbb{R}^{2}$ such that $w^{T} R_{2}(\tau, 0) x_{1}^{2}$ is strictly positive as $\tau$ varies in $\left[0, t_{0}\right]$.

Similarly, there exists $v \in \mathbb{R}^{2}$ such that $y^{T} R_{1}(T, \tau) v$ is strictly positive as $\tau$ varies in $\left[0, t_{0}\right]$.

The proof of the lemma is concluded by noticing that the pair $\left(A_{12}^{0}, A_{12}^{1}\right)=\left(v w^{T}, 0\right)$ has a nonzero image for the linear $\operatorname{map} \Psi$.

\section{Worst polynomial behavior of marginally unstable switched systems}

In this section, we generalize Theorem 10 and improve the estimate on the degree of polynomial instability recalled in (5) by providing a precise estimate of the maximal polynomial rate of divergence for a switched system on the basis of the resonance relations between subsystems. The purpose is then to estimate the sum of products appearing in (11) in the presence of resonances. In order to do so we will exploit Proposition 9. As noticed in its proof, it is possible to restate it in terms of Barabanov norms instead of Euclidean ones, that is, it is possible to replace (6) by (7). Taking $T=\bar{T}$, it is then possible to upper bound all the terms $\left\|R_{i}(j T,(j-1) T)\right\|_{i}$ appearing in (11) either by $\bar{C}$ or by 1 , the latter being possible, at $j$ fixed, only for the indices corresponding to a set of resonant subsystems. This discussion motivates the following definitions.

Definition 16. Let $r \in \mathbb{N}$ and $\bar{C} \in(0,1)$. A chord of height $r$ is a vector in $\{\bar{C}, 1\}^{r}$. The resonance degree of a chord $\gamma \in\{\bar{C}, 1\}^{r}$ is the number

$\#\left\{i \mid 1 \leq i \leq r, \gamma_{i}=1\right\}-1$.

A chord collection $\mathcal{N}$ of height $r$ is a subset of $\{\bar{C}, 1\}^{r}$. A chord collection $\mathcal{N}$ is said to be complete if, for every $\gamma \in \mathcal{N}$ and $\tilde{\gamma} \in$ $\{\bar{C}, 1\}^{r}$ such that $\tilde{\gamma}_{i} \leq \gamma_{i}$ for all $i=1, \ldots, r$, one has $\tilde{\gamma} \in \mathcal{N}$.

Two chords $\gamma, \tilde{\gamma} \in\{\bar{C}, 1\}^{r}$ do not overlap if

$\max \left\{i \mid \gamma_{i}=1\right\} \leq \min \left\{i \mid \tilde{\gamma}_{i}=1\right\}$ or

$\max \left\{i \mid \tilde{\gamma}_{i}=1\right\} \leq \min \left\{i \mid \gamma_{i}=1\right\}$. 


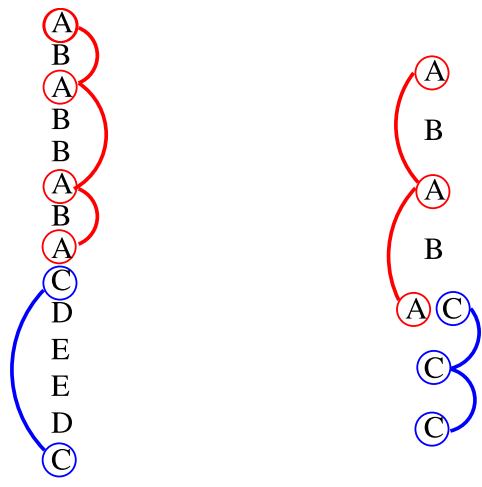

Fig. 2. Two examples of chord collections with resonance degree equal to 4 , using a compact notation; the components in resonance are represented by the same letter. Using the notations of this section, the chords of the collections are obtained by replacing 1 to the components corresponding to the same letter and setting all the other components equal to $\bar{C}$.

The resonance degree of a complete chord collection $\mathcal{N}$ is the maximum, with respect to the choice of a subset $\mathcal{N}^{\prime}$ of $\mathcal{N}$ such that no chords in $\mathcal{N}^{\prime}$ overlap, of the sum of the resonance degrees of the chords in $\mathcal{N}^{\prime}$ (see Fig. 2). More generally, the resonance degree of a chord collection $\mathcal{N}$ is defined as the resonance degree of the smallest complete chord collection containing $\mathcal{N}$.

The chord upper (resp. lower) sub-collection of height $s$ of a collection $\mathcal{N}$ is the collection of the chords formed by the first (resp. last) $s$ components of the chords of $\mathcal{N}$.

A composition $\mathcal{C}$ of length $m \leq \infty$ and height $r$ of a given chord collection $\mathcal{N}$ of height $r$ is a finite or infinite sequence $\left(\gamma^{(i)}\right)_{i=1}^{m}$ of chords of $\mathcal{N}$. Given a non-decreasing sequence $k=\left(k^{(i)}\right)_{i=1}^{m}$ of integers with $0<k^{(i)} \leq r$, the sample $\sigma_{k}$ of $\mathcal{C}$ is the sequence $\left(\gamma_{k^{(i)}}^{(i)}\right)_{i=1}^{m}$. The value of the sample $\sigma_{k}$ is the product $\Pi_{i=1}^{m} \gamma_{k^{(i)}}^{(i)}$. The value $\mathcal{V}(\mathcal{C})$ of a composition $\mathcal{C}$ is the sum of the values of all its possible distinct samples. The upper (resp. lower) sub-composition of height $s$ of a composition $\mathcal{C}$ is the composition obtained as the sequence of the chords formed by the first (resp. last) $s$ components of the chords of $\mathcal{C}$.

In the previous definition, chords represent upper bounds on the values of the terms $\left\|R_{i}(j \bar{T},(j-1) \bar{T})\right\|_{i}, i=1, \ldots, r$, in the sum (11). The choice of $\bar{T}$ is given by Proposition 9 , which guarantees that resonances can be represented by a chord containing the corresponding components equal to 1 , the other components being equal to $\vec{C}$. Thus, a composition corresponds to the choice of a switching law (each chord of the composition corresponding to a positive integer $j$ ). The value of a particular composition represents an estimate of the sum (11), while the maximum of all possible values of compositions provides a general upper bound for (11).

We are now ready to state the main technical result of this section.

Proposition 17. Let $\mathcal{N}$ be a chord collection. Then there exists a constant $K>0$ such that the value of all the possible compositions of $\mathcal{N}$ is bounded by $K\left(1+m^{L}\right)$, where $L$ is the resonance degree of $\mathcal{N}$ and $m$ is the length of the composition.

Proof. Without loss of generality, we will assume that $\mathcal{N}$ is complete. First of all, let us observe that the proposition is true when $L=0$ (Lemma 28). In the general case, the proof goes by induction on the height of the chord collection. It is clearly true when the height is equal to one (in particular there is no resonance in this case). Assume that the theorem is true for all complete chord collections of height at most $r \geq 1$. Take a complete chord collection of height $r+1$ with strictly positive resonance degree. Consider the largest $s>0$ such that the resonance degree of the

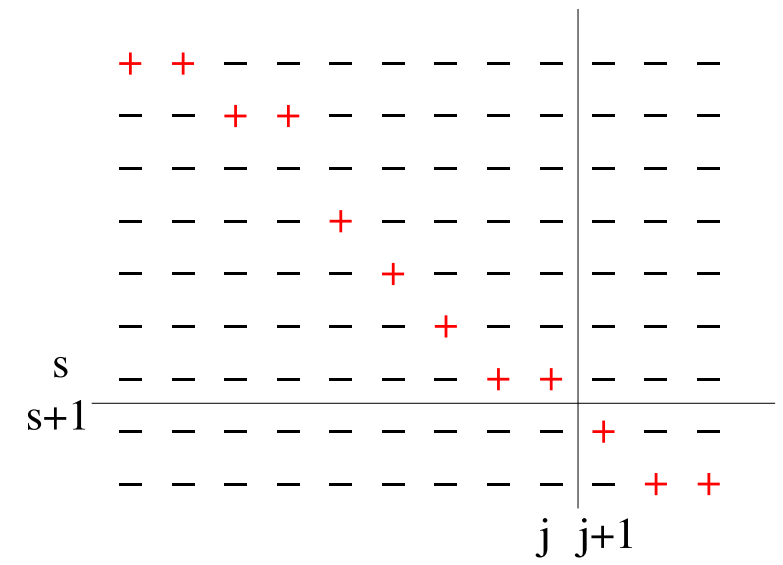

Fig. 3. One possible choice of the sample (plus signs) as in the proof of Proposition 17.

chord upper sub-collection of height $s$ is equal to zero. Then we know that the value of the upper sub-composition of height $s$ of any composition $\mathcal{C}$ is uniformly bounded by a constant $\hat{C}$. If $\hat{L} \geq 0$ is the resonance degree of the lower sub-collection of height $r+1-s$, we know by the inductive assumption that the value of the lower subcomposition of height $r+1-s$ of any composition $\mathcal{C}$ of length $\hat{m}$ is bounded by $\hat{K}\left(1+\hat{m}^{\hat{L}}\right)$, for a suitable $\hat{K} \geq 0$. Fix now $j \in\{0, \ldots, m\}$ and consider all the samples such that $\bar{k}_{j} \leq s$ and $k_{j+1}>s$ (Fig. 3 ). We deduce immediately from the previous estimates that the sum of the values of all these samples is bounded by $\hat{C} \hat{K}\left(1+(m-j)^{\hat{L}}\right)$. By taking the sum over $j \in\{0, \ldots, m\}$, we immediately obtain an estimate of the type

$\mathcal{V}(\mathcal{C}) \leq K\left(1+m^{\hat{L}+1}\right)$,

for a suitable $K>0$. Since, for some $\bar{s} \in\{1, \ldots, s\}$, there exists a chord such that the $(s+1)$-th and the $\bar{s}$-th components are equal to one, we get that the resonance degree $L$ of the chord collection satisfies $L \geq \hat{L}+1$. $^{1}$ The proposition is now proved.

Our goal is to apply Proposition 17 to the case of marginally unstable switched systems. To this purpose, we introduce the following definition.

Definition 18. Let $\mathcal{A}$ be a convex compact subset of $\mathcal{M}_{n}(\mathbb{R})$ and consider an associated maximal invariant flag of length $r$. For any family $\left\{\mathcal{A}_{i_{1}}, \ldots, \mathcal{A}_{i_{s}}\right\}$ of subsystems of $\mathcal{A}$ which are in resonance, consider the chord of length $r$ with the $i_{1}, \ldots, i_{s}$ components equal to 1 and all the other components equal to $\bar{C}$, where $\bar{C}$ belongs to $(0,1)$, and finally consider the corresponding (complete) chord collection. Then the resonance degree of the maximal invariant flag is defined as the resonance degree of such chord collection, and the resonance degree of $\mathcal{A}$ is the minimum among the resonance degrees of all maximal invariant flags associated with $\mathcal{A}$.

We are now able to state the main result of the section, Theorem 19 below, which is a straightforward consequence of Proposition 17, in view of (11).

\footnotetext{
1 Actually "=" holds. Indeed, consider a collection $\hat{\mathcal{N}} \subset \mathcal{N}$ of resonance degree $L$ containing only chords that do not overlap each other. Then it is easy to see that there exists just one chord $\gamma \in \hat{\mathcal{N}}$ and one index $i \in\{1, \ldots, s\}$ such that $\gamma_{i}=1$. We then deduce that the lower sub-collection of $\hat{\mathcal{N}}$ of height $r+1-s$ has resonance degree equal to $L-1$.
} 
Theorem 19. Let $\mathcal{A}$ be a convex compact subset of $\mathcal{M}_{n}(\mathbb{R})$ with $\rho(\mathcal{A})=0$. Let $L$ be the resonance degree of $\mathcal{A}$. Then there exists $\hat{K} \geq 1$ such that, for every solution of (1), one has, for $t \geq 0$,

$\|x(t)\| \leq \hat{K}\left(1+t^{L}\right)\|x(0)\|$.

\section{Worst polynomial behavior for discrete-time switched systems}

In this section, we derive the counterpart of Theorem 19 for discrete-time linear switched systems. Consider

$x(k+1)=A(k) x(k)$,

where $k \in \mathbb{N}, x(k) \in \mathbb{R}^{n}$, and the switching law $A(k)$ takes values in a compact set of matrices $\mathcal{A} \subset \mathcal{M}_{n}(\mathbb{R})$. The joint spectral radius $\rho_{d}(\mathcal{A})$ is defined as

$\rho_{d}(\mathcal{A}):=\lim _{k \rightarrow \infty} \max \left\{\left\|A_{i_{1}} \cdots A_{i_{k}}\right\|^{1 / k} \mid A_{i_{1}}, \ldots, A_{i_{k}} \in \mathcal{A}\right\}$.

Then system (17) is (uniformly) exponentially stable if and only if $\rho_{d}(\mathcal{A})<1$ and it admits trajectories going to infinity exponentially fast if and only if $\rho_{d}(\mathcal{A})>1$. When $\rho_{d}(\mathscr{A})=1$, two situations may occur: (i) all trajectories of (17) starting from a bounded set remain uniformly bounded and there exist trajectories staying away from the origin, in which case (17) is said to be marginally stable; (ii) (17) admits a trajectory going to infinity and the system is said to be marginally unstable.

Exactly as in the continuous-time case, one can associate with system (17) a maximal invariant flag and the corresponding block triangular matrix representations. The corresponding irreducible subsystems are unique up to reordering. Moreover, Barabanov norms exist for irreducible systems with $\rho_{d}(\mathcal{A})=1$ (cf. $[21,6]$ ). As in Remark 5, one has, for general discrete-time systems (17) satisfying $\rho_{d}(\mathcal{A})=1$, that trajectories go (possibly) to infinity at most polynomially, according to the estimate

$\|x(k)\| \leq C\left(1+k^{\hat{r}-1}\right)\|x(0)\|, \quad k \geq 0$,

where $\hat{r}$ is the number of irreducible subsystems associated with $\mathcal{A}$ with joint spectral radius equal to one (see [11]).

The resonance concept defined in Definition 6 carries over for system (17) and does not depend on the choice of a Barabanov norm. To see that, one must use a standard diagonal procedure instead of the Banach-Alaoglu theorem. Proposition 9 also holds for systems (17) with the obvious modifications, i.e., the time $\bar{T}$ is now a positive integer and the evolution operator $R_{i}\left(m_{1}, m_{2}\right)$ between times $m_{1}$ and $m_{2}$ is replaced by its discrete counterpart, namely, $R_{i}\left(m_{1}, m_{2}\right)=A_{i i}\left(m_{1}-1\right) A_{i i}\left(m_{1}-2\right) \cdots A_{i i}\left(m_{2}\right)$. Based on the discrete-time counterpart of Proposition 9, we can associate with $\mathcal{A}$ and a maximal invariant flag a chord collection and its resonance degree. The resonance degree $L_{d}$ of $\mathcal{A}$ is obtained by taking the minimum with respect to the choice of the maximal invariant flag of all such degrees. We can now state the following counterpart of Theorem 19.

Theorem 20. Let $\mathcal{A}$ be a compact subset of $\mathcal{M}_{n}(\mathbb{R})$ with $\rho_{d}(\mathcal{A})=1$. Let $L_{d}$ be the resonance degree of $\mathcal{A}$ defined as explained above. Then, there exists $\hat{c} \geq 1$ such that, for every solution of (17), one has, for $k \geq 1$,

$\|x(k)\| \leq \hat{c} k^{L_{d}}\|x(0)\|$.

The result follows from Proposition 17 and by an estimate analogous to (11). To get the latter one should notice that, using the notation $x=\left(x_{1}, \ldots, x_{r}\right)$ as in Section 3, $x_{i}(k)=\sum_{l=i}^{r} \sum_{i \leq i_{1} \leq \cdots \leq i_{k-1} \leq l} A_{i i_{1}}(k-1) A_{i_{1} i_{2}}(k-2) \cdots A_{i_{k-1} l}(0) x_{l}(0)$,

and the proof relies on the estimate of the matrix norm of

$\sum_{i \leq i_{1} \leq \cdots \leq i_{k-1} \leq l} A_{i i_{1}}(k-1) A_{i_{1} i_{2}}(k-2) \cdots A_{i_{k-1} l}(0)$.

Assume for simplicity that $k$ is a multiple of some fixed positive integer $M$, i.e., $k=q M$, then

$$
\begin{aligned}
& \left\|\sum_{i \leq i_{1} \leq \cdots \leq i_{k-1} \leq l} A_{i i_{1}}(k-1) A_{i_{1} i_{2}}(k-2) \cdots A_{i_{k-1} l}(0)\right\| \\
& \leq \hat{C} \sum_{i \leq i_{M} \leq \cdots \leq i_{(q-1) M} \leq l}\left\|R_{i}(q M,(q-1) M)\right\|_{i} \\
& \quad \times\left\|R_{i_{M}}((q-1) M,(q-2) M)\right\|_{i_{M}} \cdots\left\|R_{i_{(q-1) M}}(M, 0)\right\|_{i_{(q-1) M}},
\end{aligned}
$$

where $\hat{C}$ depends on the choice of $M$. One then simply proceeds as in the continuous-time case.

\section{Remarks on the asymptotic behavior of trajectories}

It has been shown previously that, for marginally unstable switched systems, trajectories may diverge to infinity at most polynomially, according to the estimates (16) and (20). The aim of this section is to investigate if these estimates are actually sharp. To this purpose we say that the rate of polynomial growth of a marginally unstable switched system $\mathcal{A}$ is the number $p$ satisfying

$C_{1} t^{p} \leq \max _{A(\cdot),\|x(0)\|=1}\|x(t)\| \leq C_{2}(1+t)^{p}$

for suitable positive constants $C_{1}$ and $C_{2}$ and for all $t \geq 0$.

In [12, Theorem 3] the rate of polynomial growth for marginally unstable discrete-time linear switched systems given by matrices with nonnegative integer entries has been completely characterized. This result can actually be interpreted at the light of our estimates, by observing that the number characterized in [12, Theorem 3], similarly to our resonance degree, takes into account the subsystems of the switched system which are in resonance and, additionally, the interconnection among them through suitable off-diagonal terms.

The problem of the existence of an integer $p$ and positive constants $C_{1}$ and $C_{2}$ such that (21) holds true seems to be difficult to address in full generality for marginally unstable switched linear system (it has been posed as an open question in $[7,12]$ ). However the following result, which states that the estimate of Proposition 17 is actually optimal, suggests that a candidate value for the rate of polynomial growth of a marginally unstable switched linear system is its resonance degree.

Proposition 21. Given a complete chord collection with resonance degree $L$, there exists $\hat{C}>0$ such that, for any $M \in \mathbb{N}$, it is possible to construct a composition $\mathcal{C}$ of length $M$ such that

$\mathcal{V}(\mathcal{C}) \geq \hat{C} M^{L}$.

Proof. Based on the definition of resonance degree of a complete chord collection, there exist $k \in \mathbb{N}$ and $k$ chords $\gamma^{(1)}, \ldots, \gamma^{(k)}$ in the collection such that the indices of the components equal to 1 for $\gamma^{(i)}$ are larger than or equal to those for $\gamma^{(j)}$, for $i>j$, and whose resonance degrees add up to $L$. Let $m=\left[\frac{M}{k}\right]$ and consider the composition obtained by taking $\gamma^{(1)}$ in the first $m$ chords, $\gamma^{(2)}$ in the second $m$ chords, and so on up to $\gamma^{(k)}$ from the $((k-1) m+1)$ th to the $M$-th chord. Let us count the samples of this composition with value 1: it is easy to see that their number is comparable to $m^{j_{1}} m^{j_{2}} \cdots m^{j_{k-1}}(M-(k-1) m)^{j_{k}}$, where $j_{i}$ is the resonance degree of the chord $\gamma^{(i)}$. Thus we get (22). 
The previous result motivates the following conjecture.

Conjecture 1. The rate of polynomial growth $p$ of a generic continuous-time switched system $\mathcal{A}=\operatorname{co}\left\{A^{1}, \ldots, A^{k}\right\}$ with $\rho(\mathcal{A})=0$ is well-defined and equal to the resonance degree of $\mathcal{A}$. The same conclusion holds for a generic discrete-time switched system $\mathcal{A}=$ $\left\{A^{1}, \ldots, A^{k}\right\}$ with $\rho_{d}(\mathcal{A})=1$.

Here, genericity should be understood in the spirit of Theorem 14, i.e., the diagonal blocks $A_{j j}^{l}$ are fixed for $l=1, \ldots, k, j=1, \ldots, r$, and there exists an open dense subset $\mathcal{O}$ of full measure of some suitably defined linear space so that (23) holds true if the vector made of the off-diagonal blocks of the $A^{i}$ belongs to $\mathcal{O}$. It seems reasonable that arguments adapted from the proof of Theorem 14 and Proposition 21, defining a control strategy that activates the resonant subsystems at different times, should lead to a positive answer to Conjecture 1.

Up to now we have considered global estimates on the polynomial growth of the ensemble of all trajectories. We now focus on the asymptotic behavior of single trajectories. We start our discussion with the following result.

Proposition 22. Let $\mathcal{N}$ be a complete chord collection. Then there exists a composition of $\mathcal{N}$ of infinite length such that, if $\mathcal{V}_{m}$ is the value of the composition restricted to time $m$, then

$\limsup _{m \rightarrow \infty} \frac{\log \mathcal{V}_{m}}{\log m}=L$,

where $L$ is the resonance degree of $\mathcal{N}$.

Proof. We explicitly construct the composition by concatenating compositions of increasing length of the form described in the proof of Proposition 21. We choose the lengths of these compositions recursively as follows. Assume that we have constructed the whole composition up to time $m_{i}$ and we want to complete it up to time $m_{i+1}$ by adding a composition of the form described in the proof of Proposition 21. The value of the composition up to time $m_{i+1}$ will then be larger than $\bar{C}^{m_{i}} \hat{C}\left(m_{i+1}-\right.$ $\left.m_{i}\right)^{L}$ and, for $\varepsilon>0$, up to assuming $m_{i+1}$ large enough, this is larger than $m_{i+1}^{L-\varepsilon}$. By letting $\varepsilon$ go to zero as $i$ goes to infinity, we have that

$\lim _{i \rightarrow \infty} \frac{\log \mathcal{V}_{m_{i}}}{\log m_{i}}=L$

which concludes the proof of the proposition.

The previous result motivates the following conjecture.

Conjecture 2. For a generic $\mathcal{A}=\operatorname{co}\left\{A^{1}, \ldots, A^{k}\right\}$ with $\rho(\mathcal{A})=0$, there exists a trajectory $x(\cdot)$ of (1) such that

$\limsup _{t \rightarrow \infty} \frac{\log \|x(t)\|}{\log t}=L$.

Analogously, for a generic $\mathcal{A}=\left\{A^{1}, \ldots, A^{k}\right\}$ with $\rho_{d}(\mathcal{A})=1$ there exists a trajectory of (17) such that (23) holds.

Here again, genericity should be understood as in Conjecture 1.

The next result shows that, in some cases, the asymptotic behavior of $\mathcal{V}_{m}$ is actually not comparable with $m^{L}$ for any composition of infinite length. (Note that this is not in contradiction with Propositions 21 and 22.)

Proposition 23. Let $\mathcal{N}$ be a complete chord collection and $\bar{C} \in$ $(0,1)$. Let $i$ be the largest integer such that the upper sub-collection of $\mathcal{N}$ of height $i$ has resonance degree 0 and assume that $i \bar{C}<1$. Let $\mathcal{N}^{\prime}$ be the collection made of all the chords in $\mathcal{N}$ with at least one of the first $i$ components equal to 1 . If the resonance degrees of $\mathcal{N}$ and $\mathcal{N}^{\prime}$ are different then

$\lim _{m \rightarrow \infty} \frac{\mathcal{V}_{m}}{m^{L}}=0$, for any composition of infinite length, where $\mathcal{V}_{m}$ is the value of the composition restricted to time $m$ and $L$ the resonance degree of $\mathcal{N}$. Conversely, if $i=1$ and the resonance degrees of $\mathcal{N}$ and $\mathcal{N}^{\prime}$ coincide, then (24) does not hold for some composition of infinite length.

Proof. Assume that the resonance degrees of $\mathcal{N}$ and $\mathcal{N}^{\prime}$ are different. It is clear that, for a composition of infinite length containing a finite number of elements of $\mathcal{N} \backslash \mathcal{N}^{\prime}$, the limit (24) holds. Thus, assume that the composition contains an infinite sequence of elements of $\mathcal{N} \backslash \mathcal{N}^{\prime}$. Let $n_{m}$ be the number of such elements up to time $m$. For $\varepsilon>0$, take $m$ large enough such that $(i \bar{C})^{n_{m}} \leq \varepsilon$. We want to estimate $\mathcal{V}_{T}$, the value of the composition up to time $T>m$. For this purpose, we consider all the samples such that $k_{j} \leq i$ and $k_{j+1}>i$. Since the $r-i$ lower sub-collection of $\mathcal{N}$ has resonance degree $L-1$, it is easy to see that the sum of the values of all these samples is bounded by $K^{\prime}(i \bar{C})^{n_{j}}\left(1+(T-j)^{L-1}\right)$ for some positive constant $K^{\prime}$. By taking the sum over $j \in\{0, \ldots, T\}$, we get

$$
\begin{aligned}
\mathcal{V}_{T} & \leq \sum_{j=1}^{T} K^{\prime}(i \bar{C})^{n_{j}}\left(1+(T-j)^{L-1}\right) \\
& \leq \sum_{j=1}^{m} K^{\prime}\left(1+(T-j)^{L-1}\right)+\varepsilon \sum_{j=m+1}^{T} K^{\prime}\left(1+(T-j)^{L-1}\right),
\end{aligned}
$$

which implies

$\limsup _{T \rightarrow \infty} \frac{\mathcal{V}_{T}}{T^{L}} \leq \varepsilon K^{\prime}$

and then yields (24).

Suppose now that $i=1$ and that the resonance degrees of $\mathcal{N}$ and $\mathcal{N}^{\prime}$ are equal. Without loss of generality, we can assume that the lower sub-collection of $\mathcal{N}^{\prime}$ of height $r-1$ does not have overlapping chords. We consider the composition of infinite length constructed in the proof of Proposition 22 for this sub-collection and we estimate the value of the whole composition at the times $m_{i}$. Since the first component of all the chords of $\mathcal{N}^{\prime}$ is equal to 1 , it turns out that this value coincides with the sum over $j$ of all the values of the sub-composition between times $j$ and $m_{i}$. Since $m_{i}-m_{i-1}$ is much larger than $m_{i-1}$, it is easy to see that the values of the sub-compositions are of the order of $m_{i}^{L-1}$ on a set of indices $j$ whose cardinality has order $m_{i}$. Thus, for this composition, the estimate (24) does not hold.

Remark 24. To exemplify the last part of the statement of Proposition 23, consider the case where there is just one resonance. Then, the resonance degrees of $\mathcal{N}$ and $\mathcal{N}^{\prime}$ coincide but the limit in (24) (if it exists) could be different from zero. This can be easily seen by taking the composition of infinite length containing only the chord with maximal resonance degree. The argument holds whether $i$ is equal to 1 or not. The general case where the resonance degrees of $\mathcal{N}$ and $\mathcal{N}^{\prime}$ coincide and $i>1$ seems more difficult to handle.

The above proposition implies the following result regarding trajectories of linear switched systems.

Corollary 25. Consider a linear switched system of the type (1) or (17) such that its corresponding complete chord collection $\mathcal{N}$ satisfies the conditions of Proposition 23, i.e., the resonance degrees of $\mathcal{N}$ and $\mathcal{N}^{\prime}$ are different. Then, for every trajectory $x(\cdot)$, one has

$\lim _{t \rightarrow \infty} \frac{\|x(t)\|}{t^{\Lambda}}=0$,

where $\Lambda$ is equal to $L$ or $L_{d}$. 
Note that the above result implies that the notion of polynomial instability of degree $l$ introduced in Definition 12 and applied in Theorem 14 is actually too restrictive in the general case.

We conclude this section with an explicit construction of a linear switched system of the type (1) for which the rate of polynomial growth coincides with the resonance degree and both (23) and (25) hold true. In particular, we show that the asymptotic growth of the largest norm of a point attainable at time $t$ from the unit ball does not coincide with that of any single trajectory as the time goes to infinity.

For this purpose, we consider an eight-dimensional system of the form $\mathcal{M}=\operatorname{co}\left\{M_{0}^{0}, M_{1}^{0}, M_{0}^{1}, M_{1}^{1}\right\}$

$M_{\varepsilon}^{0}=\left(\begin{array}{cccc}A^{0} & \text { Id } & 0 & 0 \\ 0 & A^{0} & \varepsilon \text { Id } & 0 \\ 0 & 0 & B^{0} & \text { Id } \\ 0 & 0 & 0 & B^{0}\end{array}\right)$,
$M_{\varepsilon}^{1}=\left(\begin{array}{cccc}A^{1} & \text { Id } & 0 & 0 \\ 0 & A^{1} & \varepsilon \text { Id } & 0 \\ 0 & 0 & B^{1} & \text { Id } \\ 0 & 0 & 0 & B^{1}\end{array}\right), \quad \varepsilon=0,1$,

where the switched systems $\mathcal{A}:=\left\{A^{0}, A^{1}\right\}$ and $\mathscr{B}:=\left\{B^{0}, B^{1}\right\}$ are irreducible and stable, i.e.,

$\rho(\mathcal{A})=\rho(\mathscr{B})=0$,

and not in resonance. Then, the resonance degree $L$ of the system is equal to 2. According to Corollary 25, (25) holds true, with $\Lambda=2$.

We choose now appropriate systems $\mathcal{A}$ and $\mathcal{B}$ and we construct switching laws illustrating that (21) is satisfied with $p=2$ and (23) holds. The construction is based on two simple lemmas (see also the comments before Proposition 3 in [12] for a related construction for discrete-time switched systems).

Lemma 26. There exist $c, T>0$ and a choice of the systems $\mathcal{A}, \mathscr{B}$ with $\rho(\mathcal{A})=\rho(\mathscr{B})=0$ and not in resonance, such that, for any unit vector $v \in \mathbb{R}^{2}$ there exists a switching law defined in $[0, T]$ whose corresponding evolution operators $R_{\mathcal{A}}(t, s), R_{\mathcal{B}}(t, s)$, associated with $\mathcal{A}$ and $\mathcal{B}$ respectively, satisfy

(i) $v$ is an eigenvector of $R_{\mathscr{B}}(T, 0)$ corresponding to the eigenvalue 1 , (ii) $\left\|\left(\int_{0}^{T} R_{\mathscr{A}}(T, s) R_{\mathscr{B}}(s, 0) d s\right) v\right\| \geq c$.

Proof. To prove the lemma one first chooses a system $\mathscr{B}$ whose worst-case trajectories are closed periodic curves of period $T$ turning around the origin (see Example 8 and $[15,17,22]$ for details). For each unit vector $v$, one can thus define a switching law such that the corresponding trajectory of $\mathscr{B}$ starting from $v$ is periodic of period $T$, so that the corresponding evolution operator $R_{\mathscr{B}}(t, s)$ satisfies (i). It is easy, for instance by slightly slowing down the dynamics of $\mathcal{B}$, to construct matrices $A^{0}, A^{1}$ arbitrarily close to $B^{0}, B^{1}$, and such that the irreducible system $\mathcal{A}$ satisfies $\rho(\mathcal{A})=$ 0 , admits closed worst-case trajectories, and is not in resonance with $\mathscr{B}$ (i.e., Condition (C1) in Theorem 14 is not satisfied). Then $\left(\int_{0}^{T} R_{\mathcal{A}}(T, s) R_{\mathcal{B}}(s, 0) d s\right) v$ can be assumed to be arbitrarily close to $\left(\int_{0}^{T} R_{\mathcal{B}}(T, s) R_{\mathcal{B}}(s, 0) d s\right) v=T R_{\mathcal{B}}(T, 0) v=T v$, uniformly with respect to the initial choice of $v$. Thus the lemma is proved for any $c<T$.

Lemma 27. Assume that $\mathcal{A}$ and $\mathcal{B}$ satisfy the conditions of Lemma 26. Then there exist positive $C, \hat{C}>0$ such that the following holds. For any $\bar{x} \in \mathbb{R}^{8}, \bar{x}=\left(\bar{x}_{1}, \bar{x}_{2}, \bar{x}_{3}, \bar{x}_{4}\right)$ with $\bar{x}_{i} \in \mathbb{R}^{2}$ there exists a trajectory $x(\cdot)$ of $\mathcal{M}$ starting from $\bar{x}$ such that

$\left\|x_{1}(t)\right\| \leq C(1+t)\|\bar{x}\| \quad$ and $\quad \limsup _{t \rightarrow \infty} \frac{\left\|x_{2}(t)\right\|}{t} \geq \hat{C}\left\|\bar{x}_{4}\right\|$.

Proof. We consider here a switching law taking values in $\left\{M_{1}^{0}, M_{1}^{1}\right\}$ and satisfying the conditions (i) and (ii) of Lemma 26 with $v=\bar{x}_{4}$. The estimate in $\left\|x_{1}\right\|$ follows basically from Proposition 17, since we are taking into account only one resonance relation. Let us compute the component $x_{2}(t)$. We have

$$
\begin{aligned}
x_{2}(t)= & R_{A}(t, 0) \bar{x}_{2}+\int_{0}^{t} R_{A}(t, s) x_{3}(s) d s \\
= & R_{A}(t, 0) \bar{x}_{2}+\int_{0}^{t} R_{A}(t, s) R_{B}(s, 0) \bar{x}_{3} d s \\
& +\int_{0}^{t} s R_{A}(t, s) R_{B}(s, 0) \bar{x}_{4} d s .
\end{aligned}
$$

We notice that the first term in the sum above is bounded. The second term is also bounded since $\mathcal{A}$ and $\mathscr{B}$ are not in resonance and because of Theorem 10. Let us consider the third term at a time $t=m T$ for a positive integer $m$. We have

$$
\begin{array}{rl}
\int_{0}^{m T} & s R_{A}(m T, s) R_{B}(s, 0) \bar{x}_{4} d s \\
= & \sum_{k=0}^{m-1} \int_{k T}^{(k+1) T} s R_{A}(m T, s) R_{B}(s, 0) \bar{x}_{4} d s \\
= & \sum_{k=0}^{m-1} R_{A}(m T,(k+1) T) \int_{k T}^{(k+1) T} \\
& s R_{A}((k+1) T, s) R_{B}(s, k T) R_{B}(k T, 0) \bar{x}_{4} d s \\
= & \sum_{k=0}^{m-1}\left[k T R_{A}(T, 0)^{m-k-1} \int_{0}^{T} R_{A}(T, s) R_{B}(s, 0) \bar{x}_{4} d s\right. \\
& \left.+R_{A}(T, 0)^{m-k-1} \int_{0}^{T} s R_{A}(T, s) R_{B}(s, 0) \bar{x}_{4} d s\right] .
\end{array}
$$

Since the modulus of the eigenvalues of $R_{A}(T, 0)$ is strictly smaller than one, the matrix

$\sum_{k=0}^{m-1} R_{A}(T, 0)^{m-k-1}$

is uniformly bounded with respect to $m$. It remains to estimate the sum $\sum_{k=0}^{m-1} k R_{A}(T, 0)^{m-k-1} \bar{w}$, where $\bar{w}=\int_{0}^{T} R_{A}(T, s) R_{B}(s, 0)$ $\bar{x}_{4} d s$ satisfies, by Lemma $26,\|\bar{w}\| \geq c\left\|\bar{x}_{4}\right\|$.

By a direct computation we have

$$
\begin{aligned}
\sum_{k=0}^{m-1} k R_{A}(T, 0)^{m-k-1}= & m\left(\operatorname{Id}-R_{A}(T, 0)\right)^{-1} \\
& +\left(R_{A}(T, 0)^{m}-\mathrm{Id}\right)\left(\mathrm{Id}-R_{A}(T, 0)\right)^{-2}
\end{aligned}
$$

proving the lemma, since $\lim _{m \rightarrow+\infty} R_{A}(T, 0)^{m}=0$.

We are ready to show, based on the previous lemmas, that the rate of polynomial growth is equal to 2 (i.e., to the resonance degree) and that (23) holds true.

To prove (21) with $p=2$ we consider the following family of trajectories starting from $\bar{x}=\left(0,0,0, \bar{x}_{4}\right) \in \mathbb{R}^{8}$ with $\left\|\bar{x}_{4}\right\|=1$. Using the previous lemma, we can choose $x(\cdot)$ such that $\left\|x_{1}(m T)\right\| \leq C(1+m T)$ and $\left\|x_{2}(m T)\right\| \geq \hat{C} m T$. We then consider a switching law taking values in $\left\{M_{0}^{0}, M_{0}^{\overline{1}}\right\}$ and such that the corresponding trajectory of $\mathcal{A}$ starting from $x_{2}(m T)$ is periodic, and we denote by $\hat{R}_{A}(t, s)$ the corresponding evolution operator. We have in particular that $\left\|x_{1}(t)\right\|=\| \hat{R}_{A}(t, m T) x_{1}(m T)+(t-m T) \hat{R}_{A}(t, m T)$ $x_{2}(m T) \| \geq \hat{c} m T(t-m T)$ for a suitable constant $\hat{c}>0$. Given any $t>0$, we consider the construction above with $m=\left[\frac{t}{2 T}\right]$ and we recover $(21)$ with $p=2$.

To construct a trajectory satisfying (23), we proceed as follows. We start from any $\bar{x}=\left(\bar{x}_{1}, \bar{x}_{2}, \bar{x}_{3}, \bar{x}_{4}\right) \in \mathbb{R}^{8}$ with $\bar{x}_{4} \neq 0$. Similarly to the previous construction, we can choose $x(\cdot)$ starting from $\bar{x}$ so that $\left\|x_{1}\left(2 t_{1}\right)\right\|=\left\|\hat{R}_{A}\left(2 t_{1}, t_{1}\right) x_{1}\left(t_{1}\right)+t_{1} \hat{R}_{A}\left(2 t_{1}, t_{1}\right) x_{2}\left(t_{1}\right)\right\| \geq$ $\hat{c}\left\|\bar{x}_{4}\right\| t_{1}^{2}$ for a suitable constant $\hat{c}>0$, if $t_{1}$ is appropriately chosen. 
The procedure can then be iterated to obtain $x\left(T_{k+1}\right)$ starting from $x\left(T_{k}\right)$, where $T_{k}=2 \sum_{i=1}^{k} t_{i}$, and the times $t_{i}$ can be chosen in such a way that

$\frac{\log \left\|x\left(T_{k+1}\right)\right\|}{\log T_{k+1}} \geq \frac{\log \left(\hat{c}\left\|x_{4}\left(T_{k}\right)\right\| t_{k+1}^{2}\right)}{\log T_{k+1}}$

converges to 2 , so that (23) is satisfied.

\section{Appendix}

Lemma 28. Let $h$ be a positive integer. Let us define

$\Xi_{m}=\left\{k \in \mathbb{N}^{h} \mid k_{1} \leq k_{2} \leq \cdots \leq k_{h} \leq m\right\}$.

Given a sequence $\alpha=\left(\alpha^{(i)}\right)_{i \in \mathbb{N}}$ in $(0,1]^{h+1}$ denote the components of $\alpha^{(i)}$ by $\alpha_{1}^{(i)}, \ldots, \alpha_{h+1}^{(i)}$. For every $k=\left(k_{1}, \ldots, k_{h}\right) \in \Xi_{m}$, let

$P(\alpha, k)=\left(\prod_{i=1}^{k_{1}} \alpha_{1}^{(i)}\right)\left(\prod_{i=k_{1}+1}^{k_{2}} \alpha_{2}^{(i)}\right) \ldots\left(\prod_{i=k_{h}+1}^{m} \alpha_{h+1}^{(i)}\right)$,

and

$S_{m}(\alpha)=\sum_{k \in \Xi_{m}} P(\alpha, k)$

Then, for any fixed $C \in(0,1)$, there exists a constant $L$ depending on $C$ such that $S_{m}(\alpha) \leq L$ for every $m \in \mathbb{N}$ and for every sequence $\alpha$ as above satisfying in addition $\alpha_{j}^{(i)} \alpha_{l}^{(i)} \leq C$ for every $j \neq l$ and every $i \in \mathbb{N}$.

Proof. Let $k^{(1)}, k^{(2)} \in \Xi_{m}$ and observe that

$P\left(\alpha, k^{(1)}\right) P\left(\alpha, k^{(2)}\right) \leq C^{\max _{l=1, \ldots, h}\left|k_{l}^{(1)}-k_{l}^{(2)}\right|}$.

Indeed, assume without loss of generality that $k_{l_{*}}^{(1)}<k_{l_{*}}^{(2)}$, where

$\left|k_{l_{*}}^{(1)}-k_{l_{*}}^{(2)}\right|=\max _{l=1, \ldots, h}\left|k_{l}^{(1)}-k_{l}^{(2)}\right|$.

Let $i$ be an integer satisfying $k_{l_{*}}^{(1)}<i \leq k_{l_{*}}^{(2)}$. If $\alpha_{j_{1}}^{(i)}$ and $\alpha_{j_{2}}^{(i)}$ are terms corresponding to the subscript $i$ in the factorization of $P\left(\alpha, k^{(1)}\right)$ and $P\left(\alpha, k^{(2)}\right)$, respectively, it is then easy to see that $j_{2} \leq l_{*}<j_{1}$, implying that $\alpha_{j_{1}}^{(i)} \alpha_{j_{2}}^{(i)} \leq C$ from the hypothesis of the lemma. Thus (26) follows.

For $q \in \mathbb{N}$, let us define the set

$\ell_{q}=\left\{k \in \Xi_{m} \mid P(\alpha, k)>C^{q}\right\}$,

and observe that, if $k^{(1)}, k^{(2)} \in \ell_{q}$, then $P\left(\alpha, k^{(1)}\right) P\left(\alpha, k^{(2)}\right)>C^{2 q}$ so that, from (26), we deduce that

$\max _{l=1, \ldots, h}\left|k_{l}^{(1)}-k_{l}^{(2)}\right|<2 q, \quad \forall k^{(1)}, k^{(2)} \in \ell_{q}$.
In particular, the set $\ell_{q}$ contains at most $(2 q)^{h}$ elements. Since $\Xi_{m}=\cup_{q=1}^{\infty}\left(\ell_{q} \backslash \ell_{q-1}\right)$, we get

$S_{m}(\alpha)=\sum_{q=1}^{\infty} \sum_{\hat{k} \in \ell_{q} \backslash \ell_{q-1}} P(\alpha, \hat{k}) \leq \sum_{q=1}^{\infty}(2 q)^{h} C^{q-1}<+\infty$.

The lemma is proved by setting $L=\sum_{q=1}^{\infty}(2 q)^{h} C^{q-1}$.

\section{References}

[1] M. Margaliot, Stability analysis of switched systems using variational principles: an introduction, Automatica J. IFAC 42 (12) (2006) 2059-2077.

[2] R. Shorten, F. Wirth, O. Mason, K. Wulff, C. King, Stability criteria for switched and hybrid systems, SIAM Rev. 49 (4) (2007) 545-592.

[3] V.D. Blondel, J. Theys, A.A. Vladimirov, An elementary counterexample to the finiteness conjecture, SIAM J. Matrix Anal. Appl. 24 (4) (2003) 963-970.

[4] V.D. Blondel, J.N. Tsitsiklis, The boundedness of all products of a pair of matrices is undecidable, Systems Control Lett. 41 (2) (2000) 135-140.

[5] L. Gurvits, Stability of discrete linear inclusion, Linear Algebra Appl. 231 (1995) 47-85.

[6] F. Wirth, The generalized spectral radius and extremal norms, Linear Algebra Appl. 342 (2002) 17-40.

[7] R. Jungers, The joint spectral radius. Theory and applications, in: Lecture Notes in Control and Information Sciences, vol. 385, Springer-Verlag, Berlin, 2009.

[8] N.E. Barabanov, An absolute characteristic exponent of a class of linear nonstationary systems of differential equations, Sibirsk. Mat. Zh. 29 (4) (1988) 12-22. 222.

[9] N.E. Barabanov, Asymptotic behavior of extremal solutions and structure of extremal norms of linear differential inclusions of order three, Linear Algebra Appl. 428 (10) (2008) 2357-2367.

[10] Z. Sun, A note on marginal stability of switched systems, IEEE Trans. Automat. Control 53 (2) (2008) 625-631.

[11] V.Y. Protasov, Fractal curves and wavelets, Izv. Ross. Akad. Nauk Ser. Mat. 70 (5) (2006) 123-162.

[12] R.M. Jungers, V. Protasov, V.D. Blondel, Efficient algorithms for deciding the type of growth of products of integer matrices, Linear Algebra Appl. 428 (10) (2008) 2296-2311.

[13] C. Curtis, I. Reiner, Representation theory of finite groups and associative algebras, in: Pure and Applied Mathematics, vol. XI., Interscience Publishers, a division of John Wiley \& Sons, New York-London, 1962.

[14] W. Liu, H.J. Sussmann, Continuous dependence with respect to the input of trajectories of control-affine systems, SIAM J. Control Optim. 37 (3) (1999) 777-803.

[15] M. Balde, U. Boscain, P. Mason, A note on stability conditions for planar switched systems, Internat. J. Control 82 (10) (2009) 1882-1888.

[16] N.E. Barabanov, Stability of two-dimensional differential inclusions, Differentsial'nye Uravneniya 26 (10) (1990) 1817-1818. 1839.

[17] U. Boscain, Stability of planar switched systems: the linear single input case, SIAM J. Control Optim. 41 (1) (2002) 89-112.

[18] A. Levine, On the stability of solutions of equations of order two, Dokl. Akad. Nauk SSSR 141 (6) (1961) 1296-1301.

[19] E. Pyatnitsky, Criterion for absolute stability of nonlinear feedback systems of order two with one nonlinear element, Autom. Remote Control 1 (1971) 5-16.

[20] N.E. Barabanov, On the Aizerman problem for a class of third-order timedependent systems, Dokl. Akad. Nauk 334 (2) (1994) 154-155.

[21] N.E. Barabanov, Lyapunov indicator of discrete inclusions, I-III, Autom. Remote Control 49 (1988) 152-157. 2823-287, 558-565.

[22] P. Mason, U. Boscain, Y. Chitour, Common polynomial Lyapunov functions for linear switched systems, SIAM J. Control Optim. 45 (1) (2006) 226-245. 